\title{
Sixth International Conference on Systems Biology (ICSB 2005)
}

The DOE grant DE-FG02-05ER64099 supported the Sixth International Conference on Systems Biology (ICSB 2005), held in Boston, Massachusetts from October 19 th to 22nd, 2005. This conference was coordinated by Prof Andrew Murray from Harvard University with help from Systems Biology institutes at other Boston area universities.

\section{Program and Speakers}

\section{Wednesday October 19, 2005}

8:00 AM - 9:00 PM Registration

9:00 AM - 5:00 PM Tutorials

6:00 PM - 7:00 PM Welcome and keynote lecture

Using fluorescence to study the E. coli chemotaxis signaling pathway

Howard Berg (Harvard University)

7:00 PM - 8:30 PM Opening Reception

Thursday October 20, 2005

8:00 AM - 5:00 PM Registration

9:00 AM Opening Remarks

Peter Sorger (Massachusetts Institute of Technology)

Session I: Biology by Design

J. J. Collins (Boston University): Integrating synthetic biology and systems biology

Christopher Voigt (University of California, San Francisco): Programming bacteria: wiring synthetic sensors and circuits to heterologous outputs

Jeffrey Tabor (University of Texas at Austin): Tuning noise in global gene expression

Jay Keasling (University of California, Berkeley): Engineering bacteria for production of an anti-malarial drug 
Lunch

$1: 30 \mathrm{PM}$

Session II: Evolution in Action

Jack Szostak (Howard Hughes Medical Institute and

Massachusetts General Hospital): Laboratory models of protocell structure and behavior

Dominique Schneider (Université Joseph Fourier):

Genotype to fitness phenotype mapping and the evolution of regulatory networks during experimental evolution in Escherichia coli

Hod Lipson (Cornell University) : Evolving engineering systems

Vitor Martins dos Santos (German Research Centre for Biotechnology): Understanding metabolic networking in microbial consortia by flux balancing, kinetic modeling and experimental validation

Joan Strassmann (Rice University): Social amoebae as model systems for genetics and evolution of social interactions

5:00 PM Keynote Lecture Steven Strogatz (Cornell University): Synchronization, singularities and circadian clocks: lessons from Art Winfree's pre-molecular approach to systems biology

6:00 - 8:00 PM Poster session and reception

\section{Friday October 21, 2005}

\section{$9: 00 \mathrm{AM}$}

Session III: Intracellular Networks

Tobias Meyer (Stanford University): Dissecting signaling networks using cell perturbations and fluorescence imaging

Peter Sorger (Massachusetts Institute of Technology): Systems biology of cytokine networks

Daphne Koller (Stanford University): Modeling conservation and variation in regulatory networks

Jörg Stelling (ETH Zürich): Precise intracellular spatial sensing through GTPase cascades 
Rama Ranganathan (Howard Hughes Medical Institute and University of Texas Southwestern Medical Center):

Evolutionary information specifying protein folding and function

Lunch

2:00 PM

Session IV: Intracellular Dynamics and

Multicellular Networks

Michael Elowitz (California Institute of Technology): Gene circuits and differentiation at the single-cell level: slow, noisy, and out of control

Eric Wieschaus (Howard Hughes Medical Institute and Princeton University): Maternal gradients and size regulation in insect embryos

Naama Barkai (Weizmann Institute of Science): Modeling threshold response to a morphogen gradient

Hiroki Ueda (RIKEN): System-level identification of mammalian circadian clocks

Claire Tomlin (Stanford University): Using mathematical modeling to help decode biological circuits

5:30 - 7:30 PM Poster session and reception

7:30 PM Student Symposium

\section{Saturday, October 22, 2005}

$9: 00 \mathrm{AM}$

Session V: Mechanics and Scale in Cellular Behavior

Julie Theriot (Stanford University): Pushing ahead: force generation and large-scale self-organization by growing actin filament networks

François Nédélec (European Molecular Biology Laboratory, Heidelberg): Simulating the interphase microtubule organization in fission yeast

Geoffrey West (Santa Fe Institute and Los Alamos National Laboratory): Universal scaling laws in biology from genomes to ecosystems: towards a quantitative 
unifying theory of biological structure and organization

Yannis Kalaidzidis (Max Planck Institute of Molecular Cell Biology and Genetics, Dresden): Rab-5 endosome movement as a regulated random walk

Mike Tyers (Samuel Lunenfeld Research Institute and University of Toronto): Cell size control networks

Lunch

$2: 00 \mathrm{PM}$

Session VI: Multicellular Networks and Intracellular Dynamics

David Harel (Weizmann Institute of Science): Comprehensive and realistic modeling of biological systems: what, how and why

Erin O'Shea (Howard Hughes Medical Institute and Harvard University): Cellular nutrient homeostasis

Mukund Thattai (National Centre for Biological Sciences, Bangalore): Dynamical properties of the yeast cell cycle network

Chao Tang (University of California, San Francisco): Anticipation and response in cell signaling

5:10 PM Keynote Lecture Bonnie Bassler (Howard Hughes Medical Institute and Princeton University): Getting the message through: reliable communication among bacteria

$6: 10$ PM Closing Remarks

Marc Kirschner (Harvard University)

Sunday October 23, 2005 and Monday October 24, 2005

9:00 AM - 5:00 PM Workshops 